\title{
LES ATELIERS DE CUISINE : UN OUTIL THÉRAPEUTIQUE POUR LE SOIN ET LE PLAISIR DES PATIENTS
}

\author{
Clémentine Hugol-Gential ${ }^{1}$, Audrey NoacCO ${ }^{2}$, \\ Christine DorlÉan ${ }^{3}$ et Sophie BetTing ${ }^{4}$
}

\begin{abstract}
Les patients atteints d'un cancer se retrouvent souvent confrontés à un ensemble de problématiques les conduisant à opérer des modifications dans leurs pratiques alimentaires. Cet article présente un dispositif d'ateliers culinaires à destination des patientes atteintes d'un cancer ainsi que son évaluation tendant à s'interroger sur son potentiel thérapeutique aidant les patients à mieux prendre en charge leur alimentation. Deux dimensions des ateliers sont plus spécifiquement étudiées ici : la déconstruction des idées reçues autour de l'alimentation et l'animation pluridisciplinaire avec la présence d'un chef de cuisine. L'exploration de ces deux points permet d'illustrer que les discours alimentaires des patients en cours de traitement se nourrissent d'un discours gastronomique autour du plaisir et de la convivialité et d'un discours médical lié à la santé et aux enjeux de soins.
\end{abstract}

1 Clémentine Hugol-Gential est maître de conférences à l'Université de Bourgogne, Laboratoire CIMEOS Lab (EA 4177).

2 Audrey Noacco est attachée à l'Université de Bourgogne.

3 Christine Dorléan est directrice des soins en oncologie médicale au Centre Georges François Leclerc à Dijon.

4 Sophie Betting est diététicienne au Centre Georges François Leclerc à Dijon.

Recherches en communication, n 48 - Article publié le 26/09/2019 


\section{Introduction}

Dans les traditions de la médecine grecque et latine, il est souvent fait allusion au rôle de la nutrition dans l'apparition de tumeurs. Mais, il a fallu attendre l'émergence de la recherche en cancérologie, au début du vingtième siècle, pour voir apparaître les premières études scientifiques visant à examiner et analyser l'influence de l'état nutritionnel sur le développement et la croissance des cancers. La question alimentaire se retrouve alors souvent au cœur des questionnements et cela pour deux raisons principales. Tout d'abord, car les pratiques alimentaires antérieures - et plus généralement l'hygiène de vie - peuvent être remises en cause par le corps médical et par les patients eux-mêmes. Mais aussi parce que les modifications sensorielles (Jakubowicz, 2006) et physiologiques induisent la mise en place de nouvelles stratégies alimentaires (Fontas, 2017).

Par ailleurs, l'arrivée de la maladie confronte le mangeur à une nouvelle identité «le mangeur-malade » (Fontas, 2017). En effet, " tomber malade » et "être admis en institution de santé » correspond à une double rupture. La première, profonde, touche la corporalité du patient qui se retrouve en prise avec une maladie qu'il doit admettre puis apprivoiser. La maladie est une rupture modifiant profondément le socle identitaire et l'altérité du malade (Hennion, 2006).

Néanmoins, la nature de la rupture corporelle liée à la maladie est protéiforme (Hugol-Gential, 2016). Cette rupture peut se traduire par une perte (sensorielle : goût, mais aussi physique : poids), des changements (physiologiques et d'habitudes) et des dégradations (physiques, physiologiques).

La seconde rupture, liée à l'entrée en institution, redéfinit entièrement l'environnement et le cadre de vie. Le patient en institution se retrouve soumis à de nouvelles règles, normes et de nouveaux rituels qui ne correspondent pas forcément à sa matrice culturelle initiale (Corbeau, 2012)

Ainsi, l'expérience alimentaire prend de nouvelles formes, s'inscrit dans de nouvelles modalités de consommation et génère la construction de nouvelles identités. Le repas est alors en ten- 
sion entre des habitudes antérieures avec une préséance de valeurs fortes de notre modèle alimentaire telles que la convivialité, le plaisir et un discours médicalisé.

Au-delà de l'expérience alimentaire en institution, se pose la question de la prise en charge alimentaire hors du contexte hospitalier, à domicile. Dans le Plan Cancer 2014-2019, 1'objectif numéro 7 est de «s'assurer des prises en charge globales et personnalisées 》 et de "permettre à chacun d'être acteur de sa prise en charge $»^{5}$ tout en privilégiant aussi une prise en charge à domicile. Le développement d'ateliers culinaires s'inscrit donc bien dans cette logique de dispositif permettant une prise en charge à domicile et une autonomie performative telle que l'entendent Sandrine Bretonnière et al. (2017) qui dans le cadre de l'évaluation d'ateliers-nutrition en région parisienne proposent ce concept pour rendre compte de l'engagement du patient dans le soin «qui va au-delà de la compliance ou de l'adhésion thérapeutique. [...] [Le patient] reste autonome dans sa capacité d'action face à la maladie, qui est étroitement liée au suivi des traitements. C'est cette performativité qu'il cherche à optimiser en améliorant ou en créant des ressources supplémentaires », Armelle Lorcy (2014) mobilise quant à elle le concept d'empowerment (pouvoir d'agir) en évoquant des ateliers d'éducation nutritionnelle : "l'éducation nutritionnelle, et plus largement thérapeutique, participe d'une volonté de promouvoir l'empowerment, "le pouvoir d'agir" et la capacité de se prendre en main des patients face au cancer et aux effets secondaires des traitements ». Dans le cadre de cet article, nous souhaitons donc interroger le dispositif d'atelier culinaire pilote développé et évalué ${ }^{6}$ afin de réfléchir à son potentiel thérapeutique. Ce type d'atelier permet-il d'accompagner le patient dans son parcours de soins ? A-t-il une valeur thérapeutique ? Quels sont les paramètres clés à déployer pour permettre au patient de mieux vivre son alimentation en situation de cancer? Il s'agit

5 https://www.e-cancer.fr/Plan-cancer/Plan-cancer-2014-2019-priorites-et-objectifs.

6 Ce dispositif fait partie du workpackage 4 « élaboration et valorisation de nouveaux dispositifs de prise en charge) du projet ALIMS (Alimentation et Lutte contre les Inégalités en Milieu de Santé) financé par l'Agence Nationale de la Recherche et le Programme National pour l'Alimentation : www.projet-alims.fr. 
alors de présenter dans un premier temps les ateliers culinaires et l'évaluation mise en œuvre grâce à une méthodologie qualitative comprenant des entretiens, des tâches de tri et un carnet de bord alimentaire réalisés par les patientes incluses dans le protocole $(\mathrm{n}=5)$ avant et après les ateliers de cuisine. Puis, de nous focaliser sur deux points qui ont émergé comme des éléments saillants dans l'animation et la conduite des ateliers : la déconstruction des idées reçues et l'animation pluridisciplinaire avec notamment la présence d'un chef de cuisine. L'exploration de ces deux points permet d'illustrer que les discours alimentaires des patients se construisent autour de deux polarités : un discours gastronomique autour du plaisir et de la convivialité qui entre en interaction avec un discours médical lié à la santé et aux enjeux de soins.

\section{La prise en charge de son alimentation en situation de cancer : le développement d'atelier de cuisine thérapeutique}

Patrice Cohen et Émilie Legrand notent que « l'alimentation est devenue à la fois un grand espoir pour la prévention des cancers - voire pour leur traitement - et à la fois une grande source d'inquiétude sur ses risques cancérigènes » (2011), Sandrine Bretonnière et al. évoquent quant à eux une "inquiétude nutritionnelle " liée notamment à une injonction de la part des médecins-oncologues, de ne pas maigrir ou de ne pas grossir. Pourtant si aujourd'hui de nombreux messages préventifs sont diffusés sur l'alimentation dans le cadre du $\mathrm{PNNS}^{7}$, il y a a contrario une place institutionnelle très faible de la prise en charge alimentaire lors de la maladie (Cohen \& Legrand, 2011) bien qu'au sein des trois Plans Cancer instaurés successivement en France il est indiqué que les patients devraient pouvoir bénéficier d'une consultation diététique (Fontas, 2017). Ceci entraîne souvent la consultation de sources dites « non conventionnelles » (Cohen \& Legrand, 2011), mais aussi de « bricolages » de la part des patients en lien

7 PNNS : Programme National Nutrition Santé. 
avec les troubles rencontrés et sources consultées (Hugol-Gential, à paraître 2019).

L'individualisation, en contexte de maladie, implique une personnalisation du soin et s'inscrit profondément dans la responsabilisation du patient face à la maladie. Ce phénomène porte l'idée de la responsabilité individuelle des choix et des pratiques alimentaires, tout comme des modes de vie en contexte de maladie. Il est important de souligner le fait que cette individualisation et cette responsabilisation, dans un contexte de cacophonie alimentaire, peuvent induire une vraie crainte de se nourrir d'autant plus que la prise en charge alimentaire est quasiment absente lors de la prise en charge du patient (Cohen \& Legrand, 2011).

En réponse à cela, on voit poindre à l'échelle nationale, des nouveaux dispositifs tels que les ateliers de cuisine thérapeutique. Ces ateliers de cuisine ont de multiples intérêts, allant du partage, à la communication en passant par la socialisation et le réapprentissage (Sidobre \& Ferry, 2000). Ils peuvent être adaptés à des individus atteints de diverses pathologies, de tout âge et ont un rôle de médiateur thérapeutique (plaisir, éveil, motricité) et de soin (estime de soi, rééducation manuelle et mnésique, etc.). Il s'agit alors de s'interroger sur leur caractère et leur potentiel en tant qu'outil thérapeutique permettant alors d'insérer ce dispositif dans le cadre d'Éducation Thérapeutique du Patient (ETP) pour une prise en charge dans le dispositif de soins. L'évaluation d'une telle démarche est alors indispensable.

Dans le cadre du dispositif pilote des ateliers culinaires développés ici, des patientes atteintes de cancer féminin, récemment diagnostiquées et ne présentant aucun trouble cognitif ont été recrutées. Au total, 5 participantes, de 29 à 65 ans, se sont engagées. Ce protocole a visé spécifiquement les femmes, celles-ci étant plus exposées aux discours médiatiques alimentaires et moins épaulées pour assurer la préparation des repas lors de leurs traitements (Mermilliod \& Mouquet, 2008). 
Tableau 1. Présentation des 5 patientes incluses dans l'étude pilote des ateliers culinaires

\begin{tabular}{|l|l|l|l|l|l|}
\hline $\begin{array}{l}\text { Nom } \\
\text { (fictif) }\end{array}$ & $\begin{array}{l}\text { Type de } \\
\text { cancer }\end{array}$ & Traitement & Âge & $\begin{array}{l}\text { Situation } \\
\text { familiale }\end{array}$ & Activité \\
\hline Alice & Utérus & Chimiothérapie & 29 ans & $\begin{array}{l}\text { Célibataire, } \\
\text { sans enfant }\end{array}$ & Pharmacienne \\
\hline Hortense & Sein & $\begin{array}{l}\text { Chimiothérapie et } \\
\text { hormonothérapie }\end{array}$ & 42 ans & $\begin{array}{l}\text { Célibataire, } \\
2 \text { enfants à } \\
\text { charge }\end{array}$ & $\begin{array}{l}\text { Responsable } \\
\text { logistique }\end{array}$ \\
\hline Nora & Sein & $\begin{array}{l}\text { Chimiothérapie et } \\
\text { hormonothérapie }\end{array}$ & 50 ans & $\begin{array}{l}\text { Célibataire, } \\
\text { sans enfant }\end{array}$ & Sans emploi \\
\hline Macha & Sein & Chimiothérapie & 56 ans & $\begin{array}{l}\text { Célibataire, } \\
1 \text { enfant à } \\
\text { charge }\end{array}$ & Kinésithérapeute \\
\hline Fantine & Ovaires & Chimiothérapie & 65 ans & $\begin{array}{l}\text { Célibataire } \\
\text { sans enfant }\end{array}$ & Retraitée \\
\hline
\end{tabular}

Un premier rendez-vous individuel a eu lieu avec chaque patiente afin de discuter de leurs pratiques et de leurs représentations alimentaires. Ce rendez-vous débutait par une tâche de tri de 22 produits alimentaires à classer librement, selon des critères propres à la participante. Une discussion enregistrée autour des critères de classifications avait ensuite lieu. Par la suite, un entretien autour des pratiques alimentaires de la participante s'est déroulé en abordant notamment les thématiques telles que le rapport à l'alimentation de la participante, son rapport à la cuisine et ses représentations de l'alimentation et du vin. À la fin de ce premier rendez-vous, un carnet alimentaire à compléter sur une durée de 7 jours a été transmis. Les participantes y indiquaient les plats préparés, les plats consommés ainsi que les éventuels ressentis lors de la préparation et/ou la consommation des plats.

La deuxième phase de cette étude pilote consistait en la mise en place des 3 ateliers culinaires. Un premier portant sur les astuces pour pallier les troubles sensoriels, un deuxième sur les astuces pour enrichir les plats et un troisième autour de la préparation de repas festifs sans se fatiguer. Ces ateliers ont été développés par l'établissement de lutte contre le cancer partenaire de cette étude 
en prenant en compte les problématiques régulièrement rencontrées par les patientes lors de leur traitement et en adéquation avec la littérature existante. Ceci a permis de définir des thématiques prédominantes explorées au sein de chaque atelier, à savoir : les troubles sensoriels, la dénutrition, la fatigue. Ces ateliers étaient organisés dans des locaux au centre-ville de Dijon hors du cadre hospitalier. Leur animation était pluridisciplinaire avec la participation de trois corps de métiers : chef de cuisine, diététicien, médecin-oncologue. À l'issue des trois ateliers, un deuxième carnet alimentaire à compléter durant 7 jours a été distribué à chaque participante afin de comparer les pratiques alimentaires avant et après les ateliers.

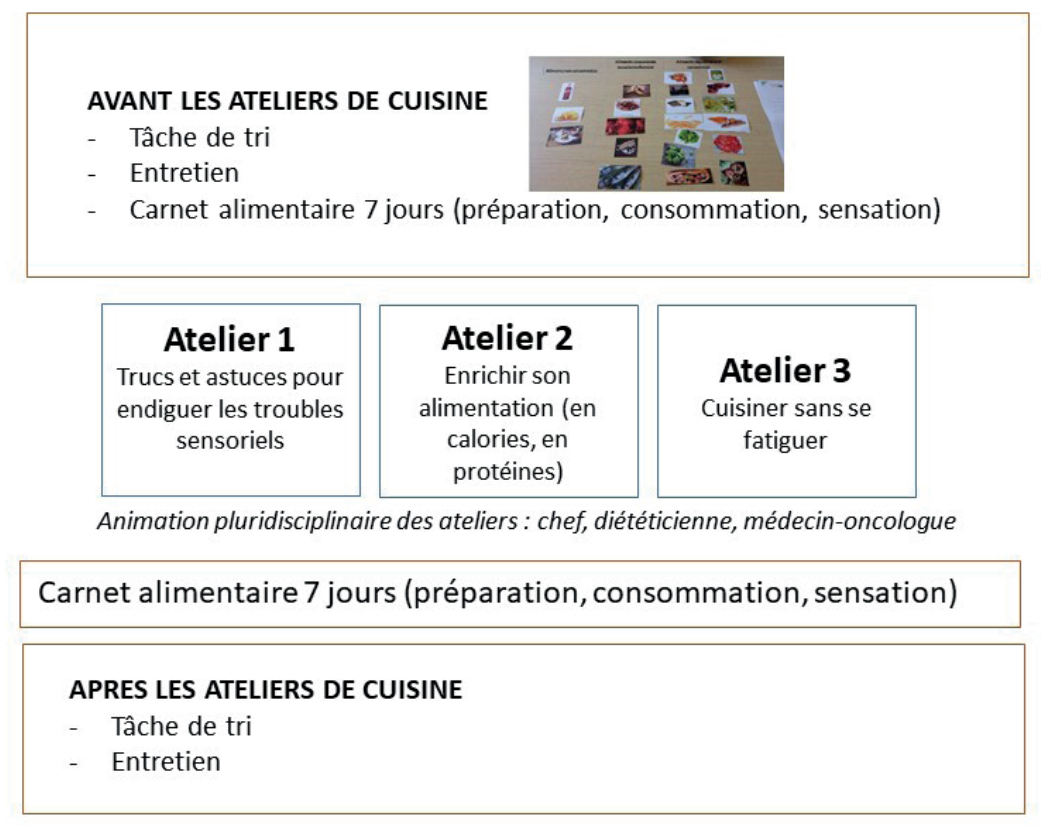

Figure 1. Panorama du protocole d'évaluation des ateliers culinaires

Un rendez-vous post-atelier a ensuite eu lieu. Le déroulement de ces rendez-vous était identique au premier (tâche de tri, discussion autour des pratiques alimentaires de la participante) et les carnets alimentaires ont été recueillis. Les thématiques abordées lors 
de l'entretien étaient les mêmes que lors du premier. L'objectif était ici de saisir s'il y avait des modifications des représentations autour de l'alimentation de la participante ainsi que son rapport à l'alimentation et à la cuisine.

Par ailleurs, la tâche de tri libre a été par la suite complétée par l'inclusion de soixante patientes atteintes de cancer (majoritairement cancer du sein) de l'établissement partenaire de lutte contre le cancer afin de mieux comprendre les stratégies de tri mises en œuvre. L'ensemble des tâches de tri et des entretiens $(n=65)$ a été enregistré pour ensuite être transcrit de manière à produire une analyse thématique.

L'évaluation des trois ateliers a mis en lumière que les participantes ne modifiaient pas fondamentalement leurs pratiques culinaires et alimentaires, mais que ceux-ci avaient néanmoins des effets bénéfiques sur deux dimensions qui sont explorées au sein de cet article : la déconstruction des idées reçues, la redéfinition du paradigme alimentaire en situation de maladie.

\section{Les ateliers de cuisine, lieu de déconstruction des idées reçues autour de l'alimentation santé}

Depuis plus de quinze ans, des campagnes d'information et d'éducation conçues par les pouvoirs publics sont menées pour faire prendre conscience aux mangeurs du lien étroit entre leur alimentation et leur santé, mais aussi pour souligner leur responsabilité individuelle dans ce domaine (Burzala-Ory, Hugol-Gential \& Boutaud, 2017). Ces campagnes d'information et d'éducation sont reprises par les « émetteurs relais » (Renaud, 2007, p. 111) que sont notamment les médias numériques et se retrouvent également largement diffusées au sein des espaces d'échanges numériques. Cette « médicalisation » de l'alimentation prend son sens dans un mouvement plus large de glissement d'une médecine thérapeutique vers une médecine plus préventive (Poulain, 2002). La santé, devenue aujourd'hui une "valeur contemporaine fondamentale » (Poulain, 2002, p. 193), recouvre donc des champs nouveaux, dont l'alimentation. 
Une littérature foisonnante autour de l'alimentation-santé et plus spécifiquement de l'alimentation-cancer émerge dans l'espace médiatique au côté d'information officielle en santé publique telle que l'OMS qui souligne qu' "une mauvaise alimentation est l'un des principaux facteurs de risque pour une série de maladies chroniques ». Pour des patients atteints du cancer la transmission et le partage d'information sont essentiels, tout particulièrement pour ce qui concerne les bonnes pratiques et les pratiques de prévention (Hugol-Gential, 2015). L'alimentation est un paramètre essentiel dans le processus de soin tant au plan physiologique qu'au plan psychologique (Hartwell et al., 2013). Dans un contexte très important de reprise en main de leur maladie et de leur situation, les patients recherchent une information fiable et rassurante sur les régimes alimentaires à adopter et/ou à privilégier (Fontas, 2017). Partager ces informations participe du processus de réassurance et de prise en charge de sa maladie, qui est une forme de résilience au niveau du patient et de ses proches. C'est pourquoi se multiplient aujourd'hui des espaces en ligne et des mises en réseau d'information que les patients consultent ou enrichissent. Santé + Magazine compte aujourd'hui 7,3 millions de fans et est l'un des médias les plus présents sur Facebook. Ce compte très actif avec plusieurs publications par jour est celui qui a généré le plus d'interactions et de discussions sur le réseau social en décembre 2017 avec des centaines de partages et des milliers de « like ». Cette page de Santé + Magazine publie très régulièrement des articles traitant de l'alimentation-cancer avec des titres du type « sept aliments qui provoquent le cancer » ou bien encore "le cancer meurt quand vous consommez ces 5 aliments, il est temps de commencer à les manger ». De plus, une littérature foisonnante « anti-cancer » émerge depuis quelques années, disséminant de nombreux conseils pour adopter une alimentation qui permettent notamment de prévenir l'apparition de la maladie (Hugol-Gential, à paraître 2019). La plupart des informations véhiculées ne reposent pas sur des études attestées et probantes ${ }^{8}$. Pour autant, les réseaux numé-

8 Voir à ce sujet le Réseau Nacre qui a publié une mise en perspective des études scientifiques réalisées sur le jeûne en France en situation de cancer : https://www6. inra.fr/nacre/Actualites/Rapport-NACRe-jeune-regimes-restrictifs-cancer-2017. 
riques ont permis une large diffusion de ces informations qui sont la plupart du temps consultées par les patients atteints de cancer, même si leur pertinence scientifique est discutable.

Depuis 2003 et la mise en œuvre des différents plans cancer en France, cette maladie a été l'objet d'une production publique et médiatique importante (Clavier \& Romeyer, 2008). Au-delà de l'impact sur le niveau d'information des patients, on constate aujourd'hui que cette multiplicité informationnelle peut avoir des effets sur les usages et les pratiques notamment concernant leur alimentation. Marine Fontas (2017) a mis en lumière que les stratégies d'adaptation alimentaires découlent très largement des informations délivrées aux patients qu'ils pourront trouver aussi bien auprès du personnel médical, des proches et des sources médiatiques.

La tâche de tri effectuée $(n=65)$ a bien souligné la mise en œuvre de nouvelles stratégies alimentaires impulsées par la patiente ou par l'entourage suite à la consultation de sources médiatiques comme en témoigne ce verbatim de patiente lors de l'entretien avant les ateliers de cuisine : "J'ai des gens qui sont venus à la maison en me disant "on a regardé! On a vu qu'il fallait que tu manges du curcuma et du gingembre". Ils m'en ont ramené. OK, donc j'en mets temps en temps dans mes plats » (Hortense. 42 ans). Parfois, la source de provenance n'est pas citée : "Ils disent qu'il faut éviter de manger du sucre parce que la cellule cancéreuse adore le sucre et donc si on évite tout ce qui est sucre lent, sucre rapide, théoriquement elle ne devrait pas se développer parce qu'elle n'a pas grand-chose à manger. J'ai entendu ce genre de chose » (M. 56 ans).

On peut souligner dans l'ensemble des cas une rupture des trajectoires alimentaires et le déploiement de différentes stratégies d'adaptations alimentaires : organisationnelle, nutritionnelle et sensorielle (Fontas, 2017). Suite à la tâche de tri effectuée auprès de nos patientes incluses dans les ateliers culinaires et ensuite auprès de 60 autres patientes, nous avons pu souligner différentes modifications alimentaires avec le retrait, l'ajout d'aliments réalisés par les patients, des modifications sur ordre médical ou bien aucune modification (Figure 2). 


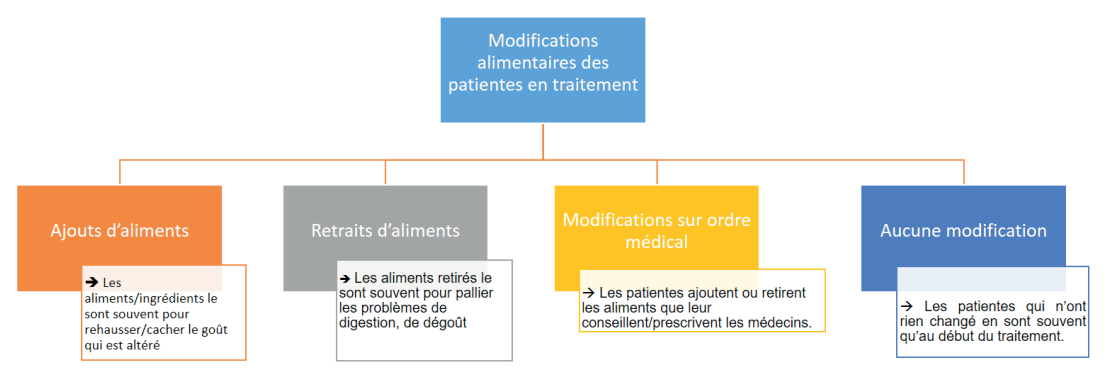

Figure 2. Les différentes modifications alimentaires réalisées par les patientes en traitement

Les ajouts et retraits d'aliments sont faits par les patients pour pallier l'émergence des contraintes physiologiques «bon j'ai été obligée de changer mon alimentation parce que j'ai beaucoup la diarrhée avec les médicaments » (Fantine, 65 ans) et sensorielles "j'ai plus d'appétit pour le salé » (Hortense, 42 ans), mais ce ne sont pas les seules raisons. En effet, l'ensemble des entretiens avec les patientes incluses dans les ateliers de cuisine ainsi que ceux réalisés lors de la tâche de tris libre montre que les femmes interrogées $(n=65)$ semblent avoir une vision plus médicalisée de l'alimentation avec des enjeux de soins sous-jacents importants. Des tensions et des ambivalences se retrouvent unies dans l'acte de manger : entre besoins physiologiques, matrices culturelles et sociales avec en écho tous les discours circulants injonctifs au niveau de l'inclusion ou de l'exclusion des aliments : " Et le gingembre et le curcuma, je sais que c'est très bon et que ce sont des bonnes épices anti-cancer. C'est la première chose qu'on m'a dite: "mange du curcuma, mange du cumin" " (Hortense, 42 ans).

Ainsi, le fait d'avoir à disposition une source pléthorique d'information peut favoriser «l'empowerment» (le pouvoir d'agir) des patients (Lorcy, 2014 ; Paganelli \& Clavier, 2014) ou bien encore l'autonomie performative (Bretonnière 2016 ; 2017) sans pour autant qu'ils aient à disposition un discours fiable et surtout en étant confronté à des discours injonctifs et contradictoires. Les patients peuvent alors s'affranchir du discours médical officiel diffusé notamment par leur médecin traitant et oncologue pour mettre en place leur propre dispositif : «[depuis la maladie] j'ai pris des 
oméga 3 si, en complément alimentaire. J'ai lu que c'était très bon pour la dépression, le moral. Comme c'est mon cas. J'avais pris ça. C'est moi qui ai décidé, pas un médecin. C'est moi qui ai réfléchi, qui fais à ma recette, à ma façon à moi ». (Nora, 50 ans).

À la lumière de l'ensemble de ces éléments, il est apparu que les ateliers culinaires ne pouvaient se contenter d'aborder uniquement les besoins nutritionnels, les modifications physiologiques, sensorielles et les techniques culinaires. Les pratiques alimentaires sont en prise avec un discours injonctif médicalisé et on note que les discours circulants ont un impact extrêmement important sur les stratégies d'adaptation alimentaire et culinaire mises en œuvre. Il s'est alors agi de penser une animation pluridisciplinaire avec une diététicienne et un oncologue pour faire écho aux différents discours à valeur scientifique consultés par les patientes et un chef de cuisine permettant de redéfinir le paradigme de l'alimentation-santé en faisant le lien avec des valeurs telles que la convivialité et le plaisir.

\section{Du soin au plaisir : de l'utilité d'une animation pluridisciplinaire}

Les stratégies de tri effectuées par les patientes $(n=65)$ soulignent différentes manières de classer et donc de se représenter son alimentation : classement par typologie d'aliment, par fréquence de consommation, par habitudes dichotomiques (j'en mange/je l'exclus de mon alimentation), par échelle hédonique (j'aime/je n'aime pas), mais aussi par un paradigme de santé opposant le sain et le non-sain, la santé et le plaisir (exemple image 1).

Les patientes, selon les contextes, l'état de santé, le traitement en cours opèrent des choix qui tendent vers une alimentation de plaisir : "Il n'y a pas de mal à se faire du bien et ce n'est pas parce que l'on est malade, qu'on doit s'arrêter encore plus de manger correctement enfin » (Hortense. 42 ans) ou, au contraire, une alimentation saine avec l'exclusion ou l'ajout de certains aliments : «Je craque des fois pour les chips, mais je sais que ce n'est pas excellent pour ma santé actuellement donc j'ai mis ça ensemble euh c'est, c'est des aliments que je ne consomme pas », "[Cur- 
cuma] j'ajoute facilement dans mon plat d'autant plus que je crois à ces vertus anti-inflammatoires »(Macha, 56 ans). La volonté d'allier les deux a beaucoup été évoquée. Au travers des discours en tension entre le médical et le plaisir, on voit une nouvelle catégorie émergée : l'alimentation bonne (au goût, pour le moral, pour le physique) qui a un impact sur le mieux-être du patient agissant sur des composantes psychologiques et physiques : « [en parlant de la place accordée à son alimentation], une grande importance ne serait-ce que pour le plaisir. Parce qu'il y a des choses que j'aime bien manger et puis après c'est sûr que cela fait des répercussions au niveau de la santé donc c'est important aussi d'avoir des choses qui sont bonnes aussi pour la santé »(Fantine, 65 ans).

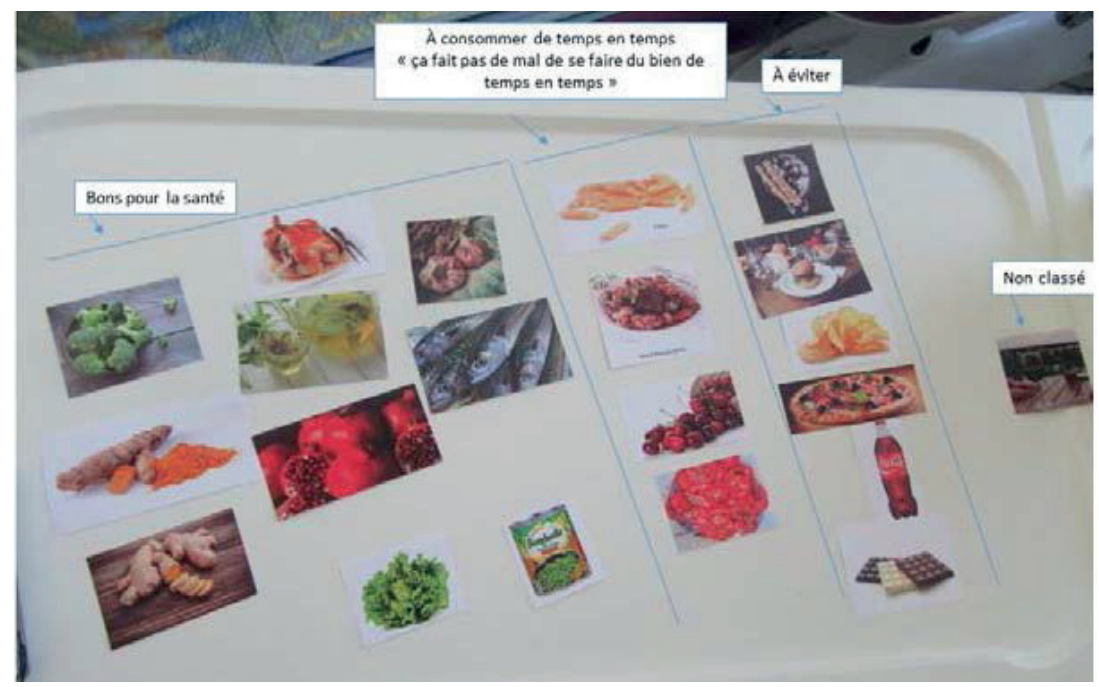

Image 1. Exemple de tri effectué par une patiente

On voit donc un triptyque se construire entre le plaisir, la santé et le mieux-être avec la mise en œuvre d'une alimentation bonne (Figure 3).

Lors des ateliers de cuisine, trois types de discours cohabitaient. Un discours médical en mobilisant des sources consultées par les patientes est discuté avec le médecin-oncologue et la diététicienne, un discours hédonique (j'aime/je n'aime pas) et un discours pratique-gastronomique. Ce dernier permet de remettre 
l'alimentation en perspective et de ne pas réduire l'acte de manger à des aspects simplement nutritionnels ou lié à des questions de santé. Le format des ateliers de cuisine et la présence du chef permettent alors de modifier le paradigme de l'alimentation-santé en remettant des dimensions au cœur de l'acte alimentaire : le plaisir et la convivialité. Ceci permet également de cultiver cette catégorie du « bon » qui n'oppose pas plaisir et santé, mais qui concilie les deux au travers notamment de pratiques culinaires spécifiques (Hugol-Gential, 2018).

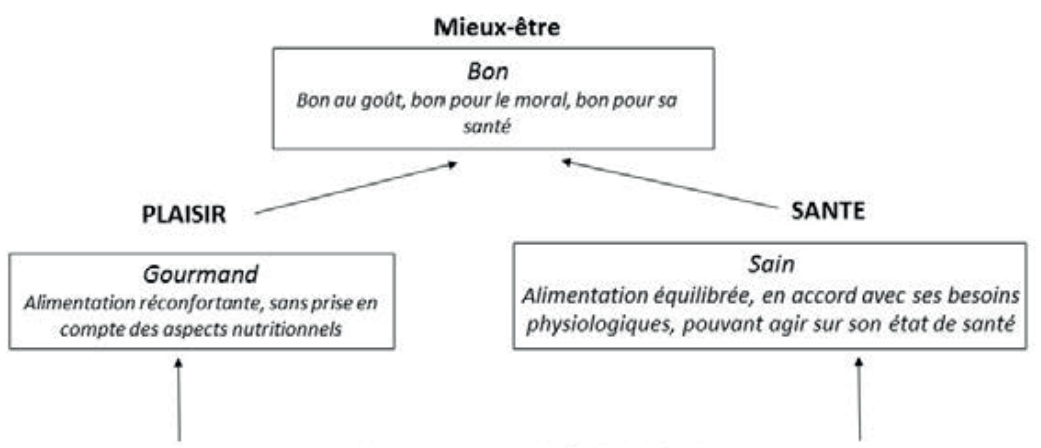

Besoins et capacités biologiques

Figure 3. Représentations et choix alimentaires selon les données de notre corpus

La présence du chef est alors un levier pour réconcilier un discours médicalisé injonctif et un discours gastronomique qui promeut le plaisir. À ce sujet, le chef Olivier Chaput ${ }^{9}$ qui anime des ateliers culinaires confiait dans une interview ${ }^{10}$ : "C'est vraiment une démarche pluridisciplinaire. Nous, nous réalisons une recette,

9 Olivier Chaput est un chef de cuisine originaire du Limousin qui s'investit dans la lutte contre le cancer. Il est à l'origine de l'Agenda des chefs dès 2011 où il fait part de 12 recettes. Par la suite, Olivier Chaput publie « 12 chefs contre le cancer, leurs meilleures recettes » regroupant de nombreuses recettes réalisées par plusieurs chefs cuisiniers connus. Les profits sont reversés au fonds de dotation « Pour Elles » dont il est le président fondateur.

10 Interview à retrouver dans Hugol-Gential, C. (Ed.) (2018). Bien et bon à manger : penser notre alimentation du quotidien à l'institution de santé. Dijon : Éditions universitaires de Dijon. 
mais en amont, nous demandons à la nutritionniste si tel ou tel ingrédient que nous souhaitons utiliser sera apprécié ou non par les patients. Elle peut très bien nous dire: "non, cet ingrédient-là, il ne vaut mieux pas, change-le avec autre chose". Finalement, nous établissons des recettes, mais ce n'est pas moi qui vais expliquer les bienfaits nutritionnels. Moi, je vais expliquer la recette et être dans le partage. C'est quelque chose de très convivial et non médicalisé. Nous ne sommes pas dans un discours injonctif non plus, mais dans une prise de conscience de son alimentation ».

Ainsi, lors des entretiens post ateliers, une des patientes confiait : "Même si je me rends compte qu'on peut manger des choses saines avec plaisir, je n'ai pas encore le réflexe » (Nora, 50 ans). Ces ateliers réalisés hors du contexte hospitalier permettent par ailleurs de dépasser la maladie, de revenir à des habitudes antérieures «c'était très bien, détendu. Et puis chaleureuse, l'ambiance assez gaie, c'était jovial. On a oublié la maladie, c'était agréable » (Macha, 56 ans), «j'aime bien faire la cuisine et j'aimais beaucoup l'idée de me retrouver avec d'autres patientes $d u$ centre et d'apprendre de nouvelles recettes, en groupe c'est toujours très enrichissant pour moi, c'est très agréable, c'est festif d'être plusieurs femmes à faire la cuisine. Ça m'a beaucoup plu cette idée-là » (Macha 56 ans). Cette dimension conviviale est également fortement valorisée dans les travaux de Bretonnière et al. (2017) sur le dispositif d'ateliers-nutrition en région parisienne : «Au travers de cette recherche de convivialité et de partage par les participants, il apparaît très nettement qu'un décrochage s'opère par rapport à la pure logique nutritionnelle originelle des ateliers ». Le format proposé permet alors de remettre l'acte culinaire en lien entre les habitudes antérieures et les habitudes qui se sont mises en place avec l'arrivée de la maladie permettant l'échange avec les soignants, les chefs et les patientes : "C'est vraiment un format qui sort de l'hôpital tout en restant dans le contexte malgré tout de la maladie, mais sans avoir la maladie placardée partout. On parle un peu de tout, de rien, on parle de la maladie forcément, mais on peut bifurquer sur : "ah moi j'ai fait ci, j'ai fait ça..." » (Hortense, 42 ans). 


\section{Conclusion}

L'acte de manger en situation de cancer se redéfinit avec la mise en œuvre de stratégies d'adaptation alimentaires : organisationnelle, nutritionnelle, sensorielle (Fontas, 2017), mais aussi en regard des différents discours circulants autour de l'alimentation-cancer. Ainsi, on voit une relation médicalisée à l'alimentation se déployer avec de forts enjeux de prévention et de soins. Le repas est alors pensé au travers d'un prisme valorisant avant tout la valeur santé en se référant notamment à des discours médicalisés utilisant une terminologie scientifique. On voit alors que le discours médical impacte le discours alimentaire dans son ensemble. Pour autant, la notion de plaisir reste très fortement présente (Dupuy, 2013) et une dichotomie prend forme opposant plaisir et santé. La réalisation des ateliers culinaires a ainsi pointé la cohabitation de différentes formes de discours et la cristallisation du fait alimentaire en situation de maladie qui se retrouve en tension entre un discours injonctif médicalisé et un discours gastronomique promouvant le plaisir.

Le cadre des ateliers culinaires permet de repenser cette opposition entre plaisir et santé et de faire le lien avec des habitudes antérieures. Ainsi, dans le dispositif développé et évalué ici, la présence d'un chef a permis notamment de remettre au cœur des ateliers un discours pratique et gastronomique et de le faire cohabiter avec des questions de santé permettant de conforter une valeur émergente, celle de l'alimentation « bonne ». Ainsi, il a été constaté que la présence d'un chef au côté de soignants (diététicienne, médecin-oncologue) permet de faire intervenir une autre terminologie et donc de remettre au cœur du discours la notion de plaisir en renforçant la catégorie du « bon » (Hugol-Gential, 2018).

Si l'évaluation des ateliers n'a pas montré de changements radicaux dans les pratiques alimentaires et culinaires des patients, elle montre néanmoins bien des enjeux liés à l'autonomie du patient avec notamment une meilleure information permettant de faire des choix éclairés quant aux pratiques alimentaires. Ceci tend alors à souligner le potentiel thérapeutique de l'atelier culinaire prenant en considération les enjeux de soins, de plaisir et de bienêtre. 


\section{Références}

Boutaud, J.-J. (2005). Le sens gourmand. De la commensalité - du goût - des aliments. Paris : Jean-Paul Rocher éditeur.

Bretonnière, S. (2016). Autonomie performative et espace de subjectivités en oncologie. Dans B. Derbez, N. Hamarat \& H. Marche (dir.), La dynamique sociale des subjectivités en cancérologie. Toulouse, Érès.

Burzala-Ory, H., Hugol-Gential, C. \& Boutaud, J.-J. (2017). L'image des légumes. Circulation des discours dans l'espace médiatique et désenchantement, Anthropology of food. Disponible à : https://aof.revues.org/8172.

Bretonnière, S., Festa, A., Bataille, P. \& Zelek, L. (2017). Apprivoiser et dépasser l'injonction de réforme alimentaire en situation de Cancer (France). Anthropology of food. Disponible à : http://journals.openedition.org/aof/8230.

Clavier, V. \& Romeyer, H. (2008). Travailler sur les discours en SIC : le cas du discours sur le cancer. Actes du congrès de la SFSIC 2008. Disponible à : http://www.sfsic. org/congres_2008/spip.php?article85.

Cohen, P. \& Legrand, E. (2011). Alimentation et cancers. Personnes atteintes et autorités alternatives. Anthropologie \& Santé, 2. Disponible à : http://anthropologiesante. revues.org/629.

Corbeau, J.-P. (2001). La filière du manger en contexte hospitalier, Face à face, regards sur la santé 3. Disponible à : http://faceaface.revues.org/565.

Corbeau, J.-P. (2012). Alimentation en milieu hospitalier. Dans J.-P. Poulain (Ed.), Dictionnaire des cultures alimentaires. Paris : Presses universitaires de France.

Dupuy, A. (2013). Plaisirs alimentaires. La socialisation des enfants et des adolescents. Coll. Table des Hommes. Rennes : Presses universitaires François-Rabelais.

Fischler, C. (2004). Le paradoxe de l'abondance. Sciences humaines 135, 22-26.

Fontas, M. (2017). Manger après le diagnostic d'un cancer. La mise en place de stratégies d'adaptations alimentaires. Anthropology of Food 12. Disponible à : http:// journals.openedition.org/aof/8244.

Hartwell, H.J., Shepherd, P.A. \& Edwards, J. S. A. (2013). Effects of a hospital ward eating environment on patients' mealtime experience: A pilot study. Nutrition and Dietetics 70(4), 332-338.

Hennion, A. (2006). Pour une approche pragmatique du soin et de l'accompagnement. Cahier de la Fondation Médéric Alzheimer, 33-36.

Hugol-Gential, C. (2015). Le repas à l'épreuve du cancer : une redéfinition sensorielle, sensible et symbolique. ESSACHESS : Journal for communication studies 8/2(16), 181-194.

Hugol-Gential, C. (Ed.). (2016). Se nourrir ou manger ? Les enjeux du repas en établissement de santé. Coll. Questions alimentaires et gastronomiques. Paris : L'Harmattan.

Hugol-Gential, C. (Ed.). (2018). Bien et bon à manger. Penser notre repas du quotidien à l'institution. Dijon : Éditions universitaires de Dijon.

Hugol-Gential, C. (2019 à paraître). Les informations autour de l'alimentation cancer : la reponsabilisation et l'expertise au cœur des discours. Questions de communication.

Jakubowicz, C. (2006). Troubles du goût et de l'alimentation chez les malades du cancer traités par chimiothérapie. Médecine et nutrition 4, 157-178. 
Lorcy, A. (2014). " Le goût de manger » pendant une chimiothérapie : difficultés et choix alimentaires des femmes traitées pour un cancer gynécologique (Québec), Sociologue et sociétés 46(2), 181-204.

Mermilliod, C. \& Mouquet, M.C. (2008). Les différents soutiens déclarés par les malades. Dans A.-G. Le Corroller-Soriano, L. Malavolti \& C. Mermilliod (Eds.), La vie deux ans après le diagnostic de cancer (pp. 321-329). Coll. Étude et Statistiques. Paris : DRESS-INSERM,.

Paganelli, C. \& Clavier, V. (2014). S'informer via des médias sociaux de santé : quelle place pour les experts? Le Temps des médias 23, 141-143.

Poulain, J.-P. (2002). Manger aujourd'hui. Attitudes, normes et pratiques. Toulouse : Privat.

Renaud, L. (2007). Les médias et le façonnement des normes en matière de santé. Québec, Presses de l'Université du Québec.

Sidobre, B. \& Ferry, M. (2000). L'atelier de cuisine : un outil thérapeutique. Soins gérontologie 21, 23-26.

\section{Remerciements}

Nous remercions vivement l'établissement de lutte contre le cancer partenaire (le Centre Georges François Leclerc de Dijon) et particulièrement Sophie Betting, (cadre de santé diététicienne) Christine Dorléan (directrice des soins), Pierre Peltre (chef de cuisine), Laure Favier et Nathalie Bremaud (médecins-oncologues) et l'ensemble de l'équipe diététique. Nous remercions le chef Grégoire Maille pour l'animation des ateliers et Anne Cazor pour son accueil dans les locaux de Scinnov. Nous adressons nos plus vifs remerciements à Alice, Hortense, Macha, Nora et Fantine pour leur participation à ce dispositif pilote. Nous remercions également les 60 autres patientes ayant participé à la tâche de tri.

Nous remercions les membres de l'équipe ALIMS et plus particulièrement Audrey Noacco, Mélanie Nallet et Marie Simon qui ont travaillé activement au sein des ateliers culinaires et de l'élaboration et de l'analyse de la tâche de tri.

Le projet ALIMS est financé par l'Agence Nationale de la Recherche (ANR) et le Programme National pour l'Alimentation (PNA).

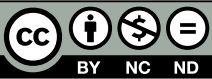

«Attribution - Pas d'Utilisation Commerciale - Pas de Modification 4.0 International» (CC BY-NC-ND) 